\title{
Sensitivity to attachment, alignment, and contrast polarity variation in local perceptual grouping
}

\author{
Louis K. H. Chan and William G. HaYwaRd \\ University of Hong Kong, Pokfulam, Hong Kong, China
}

\begin{abstract}
A number of leading theories (e.g., Grossberg \& Mingolla, 1985; Kellman \& Shipley, 1991; Rensink \& Enns, 1995) commonly assume that perceptual grouping by contour alignment occurs preattentively across reversing contrast polarity elements. We examined this notion in seven visual search experiments. We found that only grouping by attachment supported preattentive visual search and that grouping by contour alignment required attention in order to operate. Both attachment grouping and grouping by contour alignment were sensitive to contrast reversals. Further results showed that contour alignment was a strong grouping cue only among elements with the same contrast sign but that it did not facilitate grouping across reversing contrast. These results suggest that grouping by contour alignment operates only on inputs of consistent contrast polarity.
\end{abstract}

Studies of visual attention traditionally distinguish between preattentive processes that are parallel, effortless, and automatic and attentive processes that are slow, effortful, and voluntary (e.g., Broadbent, 1958; Neisser, 1967; Treisman \& Gelade, 1980). In general, preattentive processes encode primitive features from across the visual field and provide representational inputs to attentional processes. Both preattentive and attentive processes play important roles in perceptual organization, by which an observer makes sense of the units of information in the visual world. During a preattentive stage, primitive perceptual units are identified, and internal representations of these units are created. Subsequently, attentional processes may further group some of these primitive units together.

Various accounts have been offered to explain under which conditions perceptual grouping occurs preattentively and under which conditions it requires attentional resources. For instance, it is commonly assumed that perceptual grouping of properly aligned edges occurs at a low level (e.g., in the primary visual cortex) and that this mechanism is insensitive to the direction of the contrast sign at edges (e.g., Grossberg \& Mingolla, 1985; Kellman \& Shipley, 1991). These assumptions would lead to the hypotheses that perceptual grouping across aligned contours requires very little attention and that its strength is not influenced by the contrast sign of the constituent elements. Support for these hypotheses comes from phenomena such as the reverse-contrast Kanizsa square. As is illustrated in Figure 1A, the illusory square produced by grouping the opposite-polarity elements is generally perceived without much effort, and it appears as vivid as the illusory square produced by consistentpolarity elements (Figure 1B). These kinds of experiences are very much parallel to the idea that grouping by contour alignment is preattentive and is insensitive to contrast polarity.

Efforts have been made to quantify the extent to which perceptual grouping by contour alignment requires attention and is sensitive to contrast polarity. For instance, Gilchrist, Humphreys, Riddoch, and Neumann (1997) examined this issue by measuring the efficiency of visual search for targets that were perceptual groups. With this approach, since perceptual grouping is required for identifying the target, the associated search efficiency should reflect the extent to which the grouping process requires attention. Search efficiency is measured as the additional time needed when the number of distractors is increased by one. In their study, Gilchrist et al. asked participants to search for a target that was composed of two vertically aligned shapes among distractors that had the two shapes aligned horizontally. The orientation of the target was distinguished from the distractors only after their constituent shapes were grouped, so search performance reflected the efficiency of the grouping process. They found that search was fairly efficient for pairs of consistent-polarity circles $(15.6 \mathrm{msec} /$ item $),{ }^{1}$ consistent-polarity squares $(10.8 \mathrm{msec} /$ item), and opposite-polarity squares $(12.4 \mathrm{msec} / \mathrm{item})$. However, search was relatively inefficient for a pair of opposite-polarity circles $(32.2 \mathrm{msec} /$ item $)$. Therefore, grouping efficiency was impaired by reversing the contrast polarity within a group only for circles, but not for squares. They reasoned that since the squares shared collinear contours, grouping of the squares was performed by virtue of contour alignment. As such, the results were interpreted to suggest that grouping by contour alignment is insensitive to contrast polarity. Also, the fairly efficient visual search performance was interpreted to suggest that grouping by contour alignment is a preattentive process.

L. K. H. Chan, clouis@graduate.hku.hk 
A

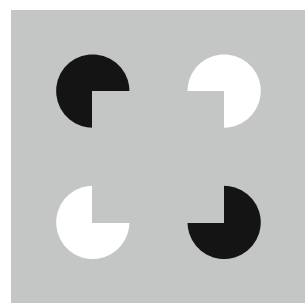

B

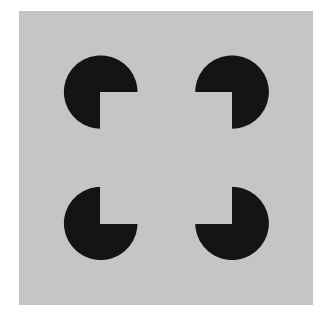

Figure 1. (A) Reverse-contrast Kanizsa figure. (B) Consistentcontrast Kanizsa figure. The illusory figures appear vivid in both cases. This phenomenon is consistent with the notion that perceptual grouping by contour alignment is insensitive to contrast polarity reversals.

More findings with regard to perceptual grouping by attachment, contour alignment, and contrast polarity were gathered in a study conducted by Rensink and Enns (1995). In their study, they carried out a number of visual search tasks with a variety of Müller-Lyer (ML) stimuli. By varying aspects of the ML elements, environments were created such that visual search depended on either individual features or overall configurations.

Figure 2 presents several sets of sample targets and distractors used in Rensink and Enns's (1995) study. In the left panel of Figure 2A, the sample target and distractor are differentiated by overall length, whereas the lengths of the horizontal lines are identical. In the right panel of Figure 2A, the objects have the same overall length, but the horizontal line of the distractor is longer than that of the target. This set of stimuli allowed Rensink and Enns to assess how robust a perceptual group would be. In the different-overall (Figure 2, left column) condition, grouping of line segments should lead to efficient visual search by differentiating overall lengths. In the differentsegment (right column) condition, however, grouping would become less useful because search should be easier by differentiating lengths of the horizontal lines, since the overall lengths were all identical. Rensink and Enns reasoned that if a group was fragile, visual attention might operate to disregard grouping, in order to allow search on the basis of the lengths of the horizontal lines. However, a group would be said to be robust if visual attention was not able to break it apart. In this case, search would become inefficient, because within-group features, including the lengths of the horizontal lines, were preempted by perceptual grouping. As such, the robustness of a grouping condition can be determined by comparing search efficiency between the different-segment and differentoverall conditions. If search was more efficient in the different-overall condition, it would mean that grouping was robust; if search was more efficient in the differentsegment condition, it would mean that grouping was fragile. Rensink and Enns found in their Experiment 1 that grouping was robust when the constituent lines were fully attached (Figure 2A), since search was more efficient in the different-overall condition. However, displays consisting of opposite-polarity lines that were detached at junction points resulted in visual search that was more efficient in the different-segment condition (Figure 2B). This showed that grouping of lines that were detached at junction points was fragile and contrasted with that for fully attached opposite-polarity lines, where more efficient search in the different-overall condition showed that grouping was robust (Figure 2C), despite reversals in contrast polarity.

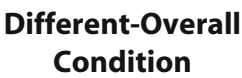

A

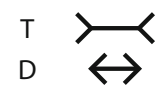

B

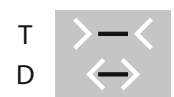

C

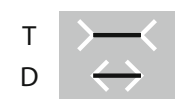

D

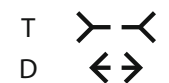

$\mathbf{E}$

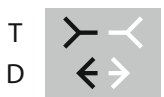

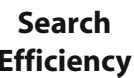

5.2

\section{Different-Segment Condition}

$\mathrm{T}$

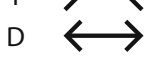

$\mathrm{T}$

D
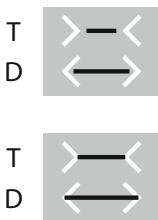

T $\prec \prec$

D $\leftarrow \rightarrow$

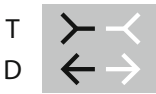

Search Efficiency

15.6

6.6

Figure 2. Stimuli and results from Rensink and Enns (1995). In the differentoverall conditions (left column), the target and the distractor differ in overall lengths but are the same in center-segment lengths. In the different-segment conditions (right column), the target and the distractor lengths are the same, but they have different center-segment lengths. Panels A-E correspond to their Experiments 1, 5E, 5A, 4A, and 5C, respectively. Please refer to the text for details. Search efficiency refers only to target-present trials, in milliseconds per item. T, target; $D$, distractor. 
Rensink and Enns (1995) used this method to study perceptual grouping by contour alignment. In their Experiment 4A (illustrated in Figure 2D), a small gap was introduced to the ML configuration. In this situation, grouping was supported by contour alignment, because the horizontal lines of the two ML wings were exactly aligned. Grouping was found to be robust, and the different-overall search was highly efficient. Performance was comparable to that of a fully attached ML configuration. They then went on to study the effect of a contrast polarity reversal across the two ML wings (Figure 2E); grouping remained robust, and the different-overall search was highly efficient when the two ML wings were separated by a small gap (it should be noted that grouping became fragile when it occurred across a large gap). These results are consistent with the common postulations that grouping by contour alignment requires little attention and is insensitive to contrast polarity.

A limitation of Rensink and Enns's (1995) method is that although the robustness of grouping could be determined, their method did not specifically assess the extent to which grouping required attention, or the grouping strength. Although it is possible to deduce the strength of grouping on the basis of different-overall search efficiency, this possibility is contingent on the assumption that different-overall search was driven primarily by differentiating the lengths of the grouped wholes. It is not clear whether this assumption holds, because individual features might also have contributed to the overall search efficiency. For instance, visual search might be facilitated by identifying the junction shapes (arrow shape or $\mathrm{Y}$ shape) of individual ML wings in contour grouping conditions (e.g., Figures $2 \mathrm{D}$ and $2 \mathrm{E}$ ). In fact, they noted that their data were also consistent with search being based on identifying the junction shapes (Rensink \& Enns, 1995, pp. 119-120). Therefore, it is not clear whether highly efficient search in their experiments should lead to the conclusion that grouping by contour alignment is preattentive. It is also unclear whether highly efficient junction-based search might overshadow any sensitivity to contrast polarity in grouping by contour alignment.

Another reason that leads us to suspect that individual features might play a role in Rensink and Enns's (1995) results is an observed inconsistency between their results and those from other perceptual grouping studies. In Rensink and Enns's study, both grouping by attachment and grouping by contour alignment supported rapid visual search up to an efficiency of about $4 \mathrm{msec} / \mathrm{item}$. In other studies, however, this efficiency was observed only in search based on grouping by attachment; search based on separated elements was generally less rapid. For example, Spehar (2002) obtained a most efficient search efficiency of $14.0 \mathrm{msec} /$ item (Control Condition D) for contour grouping conditions but a search rate of $5.6 \mathrm{msec} / \mathrm{item}$ in a corresponding attachment grouping condition (Experimental Condition B). Gilchrist et al. (1997) found that 14 out of 15 grouping conditions with separated elements had search rates of $9 \mathrm{msec} /$ item or higher, and an attachment grouping condition resulted in a search rate of $6.2 \mathrm{msec} /$ item. Elder and Zucker (1993) examined searches similar to those in Spehar's experiment and found a most efficient contour grouping search rate of $25.0 \mathrm{msec} / \mathrm{item}$ (unconnected figure condition); its corresponding attachment grouping condition had a search rate of $14.0 \mathrm{msec} / \mathrm{item}$. Across all these studies, it appears that directly attached stimuli consistently led to more efficient search than did separated stimuli. Contour groups seldom led to more efficient search than $9 \mathrm{msec} /$ item and never produced an efficiency of $5 \mathrm{msec} /$ item, although such efficiency is typical for searches for attachment groups. In Rensink and Enns's study, however, contour groups supported a search efficiency that was directly comparable to that supported by attachment groups. In our view, one potential reason behind this inconsistency was that searches in Rensink and Enns's study might have been driven partially by individual features.

The aim of the present study was to reevaluate how the strength of perceptual grouping is affected by attachment, alignment, and contrast polarity. A straightforward expectation is that contour grouping would require little attention and would be insensitive to contrast polarity. This result would be consistent with the studies of Rensink and Enns (1995) and Gilchrist et al. (1997) and would provide an empirical basis for models of perceptual grouping that rely on these assumptions. However, it remains possible that the results of these studies were due to particular characteristics of the experimental methods used. Therefore, we investigated a number of conditions that were similar to those examined in Rensink and Enns's study, while carefully avoiding individual features that could contribute to visual search. Specifically, we reconstructed distractor stimuli that were isolated halves of the corresponding target stimuli (i.e., scattered individual ML wings). As such, if the target was perceptually grouped, it would have an effective length at least double that of the corresponding distractor, which would lead one to expect higher search efficiency than in Rensink and Enns's different-overall conditions. However, the target would not be distinguished from two distractors if they were not perceptually grouped. Search would be inefficient in this case, because serial checking for a complement of each individual ML wing would be needed. Therefore, since grouping was necessary for search, search efficiency could directly represent the efficiency of grouping in each condition.

\section{EXPERIMENT 1}

Before beginning the experiments, it was important to first test whether our stimuli could produce robust contour grouping in terms of Rensink and Enns's (1995) criterion, because we planned to use a distinction between targets and distractors that was different from theirs in subsequent experiments. In Experiment 1, we attempted to replicate Rensink and Enns's Experiment 5C, in which they used paired ML wings of opposite polarity, separated by a small gap, and tested a different-overall condition and a different-segment condition. They found a robust group pattern of performance (more efficient search in the 
different-overall condition than in the different-segment condition). Since they found that such grouping became fragile when the gap was too large (Experiment 5D), a replication of Experiment 5C would also allow us to justify that our gap size was appropriate, since our tests focus around short-range grouping. Therefore, if we replicated their results with our stimuli, our subsequent experiments that were based on these stimuli could be said to be fair tests of Rensink and Enns's findings.

\section{Method}

Participants. Twelve students from the Chinese University of Hong Kong participated in this experiment. All had normal or corrected-to-normal vision. They were paid HK $\$ 50$ for a 1-h session. One participant was replaced due to an exceptionally high variation in response time (RT).

Apparatus and Stimuli. The stimuli were presented on a computer display with a gray background. The participants viewed the visual search display from a distance of approximately $57 \mathrm{~cm}$. The stimuli were constructed to mimic Rensink and Enns's (1995) Experiment $5 \mathrm{C}$. The stimuli were all full ML configurations, in which a target was composed of two Y-wings and a distractor was composed of two arrow-wings. A long ML configuration with Y-wings was used as the target in both different-overall and different-segment conditions, and short and long ML configurations with arrow-wings were used, as distractors in the different-overall and different-segment conditions, respectively (see Figure 3 for an illustration). The overall length of a short item was $1.4^{\circ}$ (visual angle), and the overall length of a long item was $2.1^{\circ}$. Both long and short items had a gap size of $0.47^{\circ}$. The mapping of contrast polarity (black or white) to each side of each item was fixed within a block, manipulated between blocks. Three set sizes $(4,9$, and 16$)$ were used. The stimuli were distributed in invisible cells that formed a circular region with a radius of $11.7^{\circ}$ centered in the middle of the display. ${ }^{2}$ Each cell contained one item, and each item was never closer than $1.36^{\circ}$ to any other.

Design. There were three within-subjects factors - namely, set size $(4,9$, or 16), target presence (present or absent), and test condition (different overall or different segment). The experiment consisted of four blocks, and each block tested a combination of a test condition and a direction of reversing contrast polarity across the two sides of each item. Set size and target presence were manipulated within a block. Each block involved 90 trials, which were immediately preceded by an additional 15 practice trials, although the participants were not informed that these trials were for practice. The order of the four blocks was determined according to a Latin square. The first block for each participant was repeated once; this additional block served as a practice for the visual search task in general.

Procedure. The participants were asked to respond quickly and accurately. At the beginning of each trial, a fixation cross (defined with uniform black and white dots) was shown in the center of the

\section{Different-Overall Condition}

A

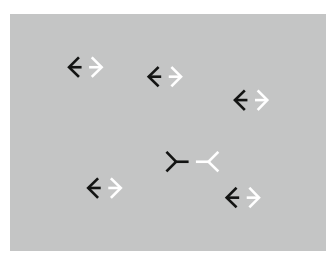

Different-Segment Condition

B

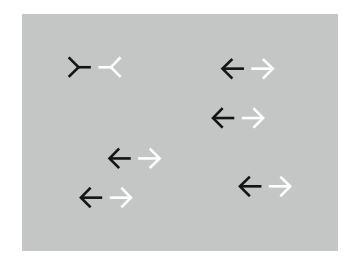

Figure 3. Illustration of search displays in Experiment 1, a replication of Rensink and Enns's (1995) Experiment 5C. (A) Different-overall condition. (B) Different-segment condition. display for a duration, chosen randomly, from 833 to $1,167 \mathrm{msec}$ long. After a blank interval of $50 \mathrm{msec}$, a visual search display was shown until a response was made or $10 \mathrm{sec}$ had elapsed. The participants were instructed to respond with a computer mouse. The left button was pressed by the left hand for target-present trials, and the right button was pressed by the right hand for target-absent trials. After the completion of a trial, an intertrial interval of $500 \mathrm{msec}$ occurred.

\section{Results}

Trials with RTs shorter than $200 \mathrm{msec}$ and the $1 \%$ of trials with the longest RTs were excluded from the analysis, as were incorrect responses. Linear regressions were carried out on RTs for each participant, and the search efficiency was obtained. The results are shown in Figure 4. A two-way repeated measures ANOVA was performed, with search efficiency as the dependent variable and target presence and condition as independent variables. The results showed that search was more efficient for targetpresent than for target-absent trials $[F(1,11)=5.62, p<$ $.04]$ and was more efficient for different-overall than different-segment trials $[F(1,11)=39.98, p<.001]$. The interaction between target presence and condition was not significant $[F(1,11)=1.38, p>.2]$. Error rates were low ( $2.5 \%$ of the trials) and showed no trend toward a speedaccuracy trade-off.

Because it is less straightforward to interpret targetabsent RTs (e.g., Chun \& Wolfe, 1996), we also analyzed the target-present trials on their own. A paired $t$ test confirmed the combined analysis that different-overall search was more efficient than different-segment search $[t(11)=$ $3.36, p<.01]$.

\section{Discussion}

In this experiment, we successfully replicated Rensink and Enns's (1995) Experiment 5C, and we found that search was more efficient in the different-overall condition $(3.6 \mathrm{msec} / \mathrm{item})$ than in the different-segment condition $(9.2 \mathrm{msec} /$ item $)$. The magnitudes of search efficiency were also comparable with theirs $(4.0$ and $13.8 \mathrm{msec} /$ item, respectively; see Figure 2E). With our stimuli, we found the same pattern of results as that in their study, that contour grouping across contrast polarity was robust and highly efficient. The present results ensured that our stimuli had the appropriate gap size and allowed us to use these stimuli in our other experiments.

\section{EXPERIMENT 2}

In Experiment 2, we examined the first focus of our investigation: whether contour grouping is a preattentive process. We asked participants to search for an ML configuration among its broken halves (i.e., individual ML wings), so that search would be possible only if each half of the target was perceptually grouped as a whole. This method allowed search efficiency to be safely interpreted as grouping strength. We created four conditions in which to examine the contribution from contour alignment in perceptual grouping (see Figures 5A-5D for illustrations). In Condition A, the participants searched for an attachment group (a fully attached ML configuration) among 
Target-Present Trials

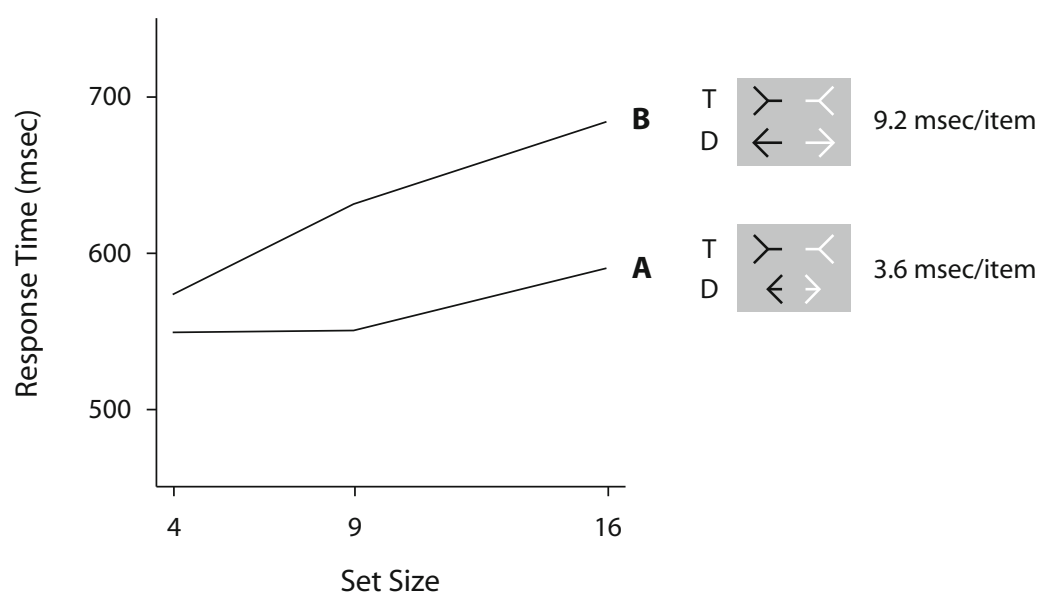

Target-Absent Trials

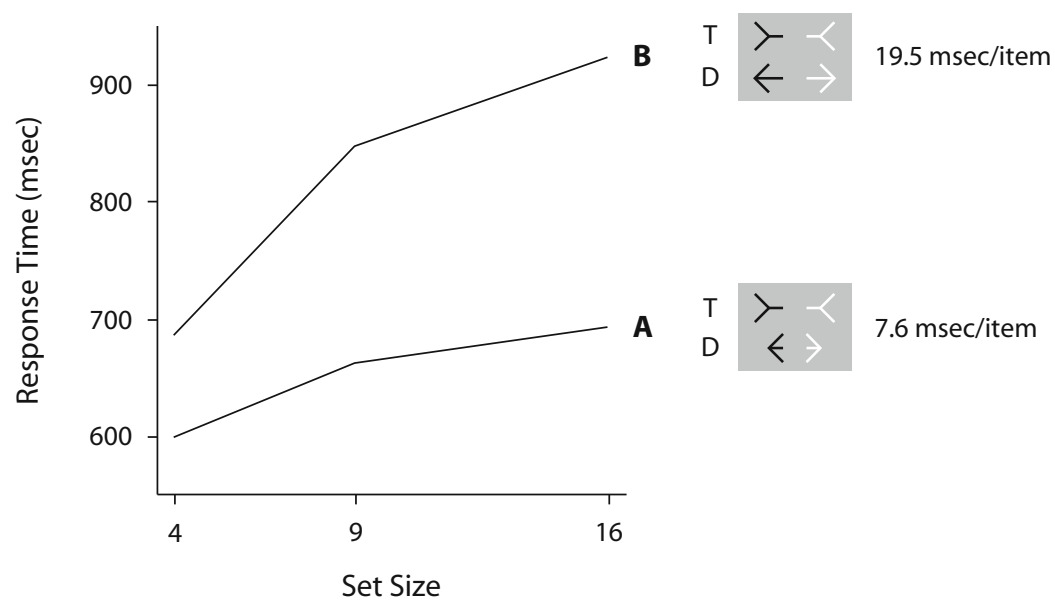

Figure 4. Results of Experiment 1. Search was more efficient in the different-overall condition (A) than in the different-segment condition (B), replicating Rensink and Enns's (1995) results. T, target; D, distractor.

individual ML wings. In previous studies, grouping of directly attached lines was found to be preattentive, and so we expected search in this condition to be highly efficient. In Condition B, the participants searched for a contour group (an aligned pair of ML wings) among individual ML wings. By comparing search efficiency in this condition with that in Condition A, we could assess the extent to which contour grouping required attention. In Condition $\mathrm{C}$, a pair of ML wings was jittered vertically so that the line segments at each side of the gap were no longer aligned. The participants searched for a misaligned group among individual $\mathrm{ML}$ wings. By comparing this condition with Condition B, we could assess the extent to which contour alignment contributed to grouping strength, because this condition was identical to Condition B except for the misalignment. In Condition D, we tested whether contour grouping occurred automatically. We created a situation in which the participants searched for an attachment group among contour groups. In this situation, the occurrence of contour grouping was not useful, because search would be easier with each contour group (with an overall length identical to the target) broken down into two individual ML wings (with shorter lengths than the target). Therefore, if contour grouping occurred automatically, search would be inefficient; however, if no grouping occurred, search should be as efficient as in Condition A.

\section{Method}

Participants. Eight students from the Chinese University of Hong Kong participated in this experiment. All had normal or correctedto-normal vision. They were paid HK $\$ 25$ for a half-hour session. One participant was replaced due to a high error rate.

Apparatus and Stimuli. The apparatus involved was the same as that in Experiment 1. The stimuli were based on those in Experiment 1 (see Figure 5 for an illustration of the search displays in each condition). In Condition A, the target was a fully connected ML configuration. In Condition B, the target was an aligned pair of ML wings. In Condition $\mathrm{C}$, the target was a misaligned pair of ML wings, with the left wing downward jittered by $0.28^{\circ}$. In these conditions, the distractors were individual ML wings $\left(0.82^{\circ}\right.$ long). In Condition D, 
A

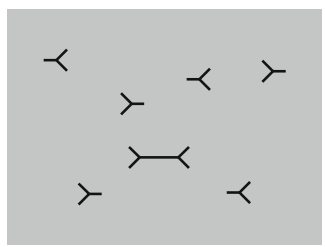

C

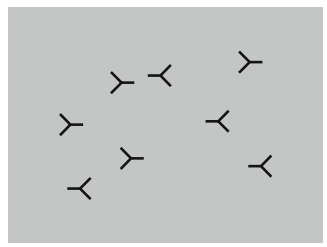

B

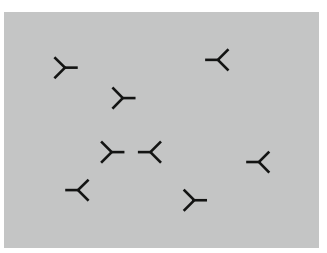

D

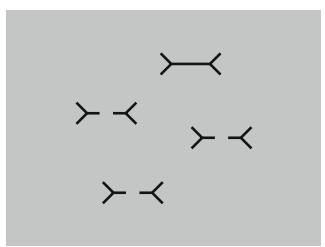

Figure 5. Illustration of search displays in Experiment 2. (A) Search for an attachment group among individual ML wings. (B) Search for a contour group among individual ML wings. (C) Search for a misaligned group among individual ML wings. (D) Search for an attachment group among contour groups.

the target was a fully connected ML configuration, and the distractor was an aligned pair of ML wings. In the search display, the background was gray, and the figures were either all black or all white within a trial. The contrast signs were mixed across trials. The set size of a display was defined as the total number of ML wings, instead of the total number of search items, because the distractor item in Condition $\mathrm{D}$ would be equivalent to two individual ML wings if it was not perceptually grouped. For a fair comparison with Condition A, across all conditions in this experiment, each full ML configuration was considered as two ML wings in calculating the set size.

The stimuli were then distributed in invisible cells defined in the same manner as in Experiment 1. For set sizes of 4, 10, and 16, in each trial, two, five, and eight cells were chosen among all possible cells. Either one ML configuration or two individual wings would be assigned to each chosen cell. Items were randomly located within a cell, but no two items were closer than $0.97^{\circ}$.

Design. There were three within-subjects factors-namely, set size $(4,10$, or 16), target presence (present or absent), and test condition. Four blocks were defined; each test condition constituted a block. Target presence and set size were manipulated within a block. Each block involved 120 trials, which were immediately preceded by an additional 15 practice trials. The blocks were ordered according to a Latin square.

Procedure. The procedure was generally identical to that in Experiment 1 , except that the fixation cross was shown for a duration, chosen randomly, of 500-1,500 msec.

\section{Results}

The data were screened and search efficiency was obtained as in Experiment 1. The results are shown in Figure 6. A two-way repeated measures ANOVA was performed, with search efficiency as the dependent variable and target presence and test condition as independent variables. The results showed that search was more efficient for target-present trials $[F(1,7)=12.75, p<.01]$, search efficiency differed across test conditions $[F(3,21)=$ $17.51, p<.001]$, and the interaction between target presence and test condition was significant $[F(3,21)=5.23$, $p<.01]$. As can be seen in Figure 6, the interaction was mainly due to a target-present/target-absent search efficiency ratio and did not reflect different patterns of performance with the target being present or absent. Error rates were low (1.6\% of the trials) and showed no trend toward a speed-accuracy trade-off.

Pairwise comparisons were conducted to compare the three pairs of conditions $(\mathrm{AB}, \mathrm{BC}$, and $\mathrm{AD})$ that we were interested in. We analyzed both the combined data of target-present and target-absent trials and the data of target-present trials only. The results showed that search was more efficient for an attachment group than for a contour group [combined, $F(1,7)=29.00, p<.01$; present only, $F(1,7)=5.05, p=.06$ ], search was more efficient for a contour group than for a misaligned group [combined, $F(1,7)=12.04, p<.02$; present only, $F(1,7)=$ $5.77 p<.05]$, and search for an attachment group was less efficient among contour groups than among individual ML wings [combined, $F(1,7)=16.38, p<.01$; present only, $F(1,7)=48.58, p<.001]$.

\section{Discussion}

In this experiment, search for an attachment group was found to be highly efficient ( $3.8 \mathrm{msec} /$ item), suggesting that a preattentive grouping process is responsible for grouping by direct attachment. This result is consistent with most previous findings. Search for a contour group was fairly efficient $(9.2 \mathrm{msec} / \mathrm{item})$. This result is consistent with the general finding that grouping by contour alignment requires relatively few attentional resources. However, the fact that a contour group supported less efficient search than did an attachment group suggests that contour grouping requires at least some attention to operate and is not a completely preattentive process. Search was more efficient for a contour group than for a misaligned group (16.3 msec/item). This suggests that contour alignment significantly facilitated perceptual grouping. In addition, we found that contour grouping occurred automatically; even though search should be easier without grouping in Condition D, a lower efficiency $(9.5 \mathrm{msec} /$ item) than in Condition A showed that some grouping occurred for the paired ML wings distractors.

In this experiment, we found that contour alignment contributed significantly to facilitating perceptual grouping and that contour grouping required only a small amount of attentional resources. These findings are generally consistent with traditional suggestions. However, we found that search efficiency differed in magnitude from that in comparable conditions in Rensink and Enns's (1995) study. For attachment grouping (Condition A), search efficiency was comparable between our study ( $3.8 \mathrm{msec} /$ item) and Rensink and Enns's study (Experiment 1A: $5.2 \mathrm{msec} /$ item). However, discrepancies arise for contour grouping search (Condition B, 9.2 msec/item; Rensink \& Enns Experiment 4A, $3.1 \mathrm{msec} /$ item) and misaligned grouping search (Condition C, $16.3 \mathrm{msec} /$ item; Rensink \& Enns Experiment 6A1, $2.7 \mathrm{msec} /$ item). These discrepancies suggest that Rensink and Enns's measurements might be at least partially influenced by isolated junction shapes.

\section{EXPERIMENT 3}

In Experiment 3, we addressed the second focus of our investigation: whether grouping by contour alignment and/ 
Target-Present Trials

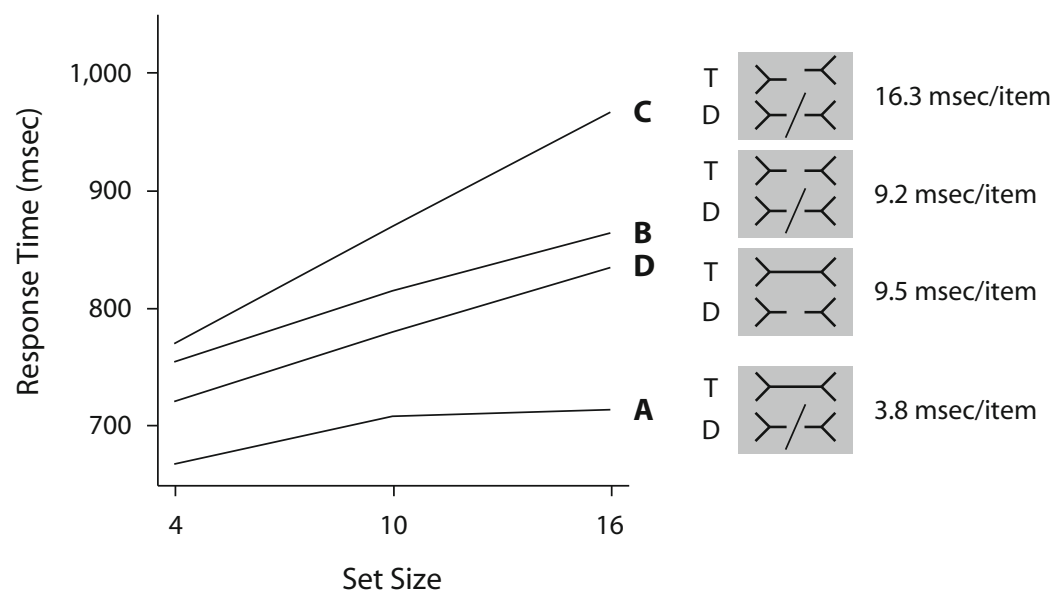

Target-Absent Trials

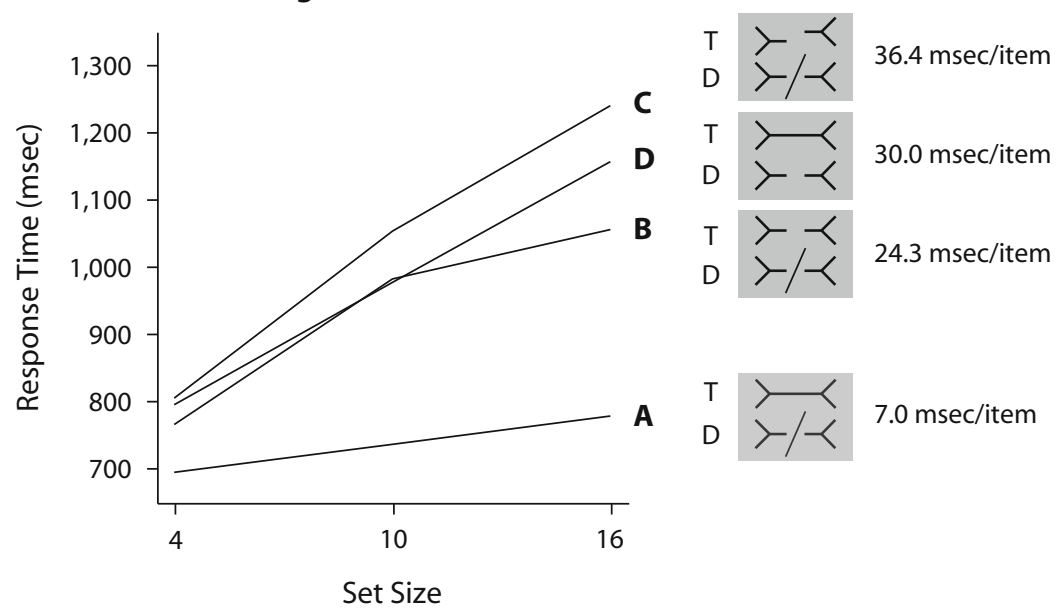

Figure 6. Results of Experiment 2. Search was more efficient for an attachment group (A) than for a contour group (B), which was, in turn, more efficient than for a misaligned group $(C)$. These results show that contour alignment facilitates grouping, but it requires more attention than does attachment grouping. Search was fairly efficient for an attachment group among contour groups (D). Please refer to the text for further details. T, target; $D$, distractor.

or attachment is sensitive to contrast polarity. Previously, Rensink and Enns (1995) found that attachment grouping was robust and supported highly efficient search, regardless of changes in contrast polarity. Spehar (2002) also found that when supported by perceptual closure, grouping by attachment was insensitive to contrast polarity, at least when a polarity reversal did not occur at a junction point. In this experiment, we introduced a contrast polarity reversal to an ML configuration target by assigning opposite contrast polarity for its left and right ML wings.

We designed six conditions (illustrated in Figures 7A$7 F)$ to examine the issues above. First, we measured search efficiency for an attachment group (Condition A) and a contour group (B) of a consistent contrast polarity. The measurements could serve as a baseline for comparisons with Conditions $\mathrm{C}$ and $\mathrm{D}$, in which search efficiency was measured for opposite-polarity attachment and contour groups. Comparisons between Conditions $\mathrm{A}$ and $\mathrm{C}$ and between Conditions B and D therefore reflected the extent to which attachment grouping and contour grouping were sensitive to contrast polarity. If similarly efficient search were to be found between consistent- and opposite-polarity groups, it would mean that grouping was not sensitive to the contrast polarity of the constituent parts. However, if grouping processes selected inputs from a single contrast sign, less efficient search would be expected for Conditions $\mathrm{C}$ and $\mathrm{D}$ than for Conditions $\mathrm{A}$ and $\mathrm{B}$.

In Condition $\mathrm{E}$, we examined a condition in which an opposite-polarity group was misaligned and, therefore, grouping was not supported by contour alignment. If contour grouping required inputs of consistent contrast polarity, search performance in this condition should be 
similar to that in Condition $\mathrm{D}$, because grouping would not benefit from contour alignment in either case. However, if search was less efficient in this condition than in Condition D, this would mean that contour grouping still occurred to facilitate search, despite the reversing contrast polarity.

In Condition F, we examined whether there was any grouping operating automatically across an oppositepolarity contour group. The method and the rationale in this condition were similar to those in Experiment 2D. A less efficient search performance in this condition than in Condition $\mathrm{C}$ would mean that aligned opposite-polarity elements were automatically grouped.

\section{Method}

Participants. Twelve students from the University of Hong Kong participated in this experiment. All had normal or corrected-tonormal vision. They were paid HK $\$ 40$ for a 40 -min session.

Apparatus and Stimuli. Search displays for each condition are illustrated in Figure 7. The settings for the apparatus and the positioning of stimuli were based on Experiment 2. In Conditions A and $\mathrm{B}$, contrast polarity was consistent within a group. Search items in the same trial were either all black or all white, determined randomly for each trial. In Condition A, the target was a fully connected ML configuration, and the distractors were broken halves of that configuration, which were longer versions $\left(1.1^{\circ} \mathrm{long}\right)$ of the individual ML wings that had been used in the previous experiments. In Condition B, the target was a pair of aligned ML wings, and the distractors were individual ML wings of the same length as in previous experiments $\left(0.82^{\circ}\right)$. In Conditions $\mathrm{C}-\mathrm{F}$, reversing contrast polarity
A

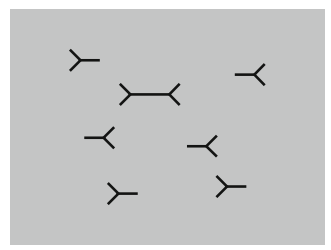

C

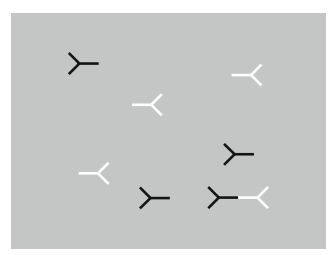

E

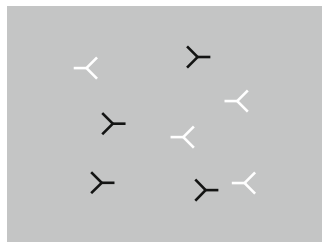

B

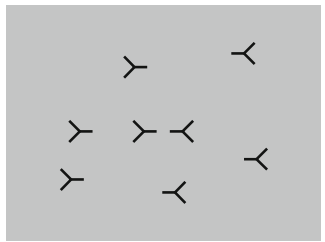

D

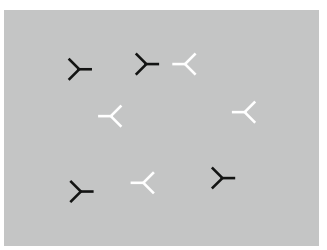

$\mathbf{F}$

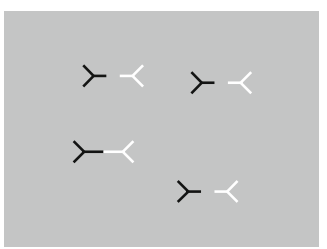

Figure 7. Illustration of search displays in Experiment 3. (A) Search for an attachment group among individual MüllerLyer (ML) wings. (B) Search for a contour group among individual ML wings. (C) Search for an opposite-polarity attachment group among individual ML wings. (D) Search for an oppositepolarity contour group among individual ML wings. (E) Search for an opposite-polarity misaligned group among individual ML wings. (F) Search for an opposite-polarity attachment group among contour groups. was assigned to the left and right halves of each ML group and to the left- and right-facing individual ML wings. Within a trial, the mapping between contrast polarity and the direction of ML wings was fixed, and this mapping was determined randomly for each trial. In Condition C, the target was a fully connected ML configuration. The distractors were broken halves of the target stimulus (i.e., long ML wings). In Condition $\mathrm{D}$, the target was a pair of aligned ML wings, and the distractors were individual ML wings. In Condition E, the target was a pair of vertically jittered $\left(0.28^{\circ}\right) \mathrm{ML}$ wings, and the distractors were individual ML wings. In Condition F, the target was a fully connected ML configuration, and the distractor was a pair of ML wings.

Design. The design of the experiment was identical to that in Experiment 2 (except that there were six test conditions).

Procedure. The procedure was identical to that in Experiment 2.

\section{Results}

The data were screened and search efficiency was obtained as in Experiment 1. The results are shown in Figure 8. A two-way repeated measures ANOVA was performed, with search efficiency as the dependent variable and target presence and test condition as independent variables. The results showed that search was more efficient for targetpresent trials $[F(1,11)=28.67, p<.001]$, search efficiency differed across test conditions $[F(5,55)=21.73$, $p<.001]$, and the interaction between target presence and test condition was significant $[F(5,55)=4.41, p<.01]$. As can be observed in Figure 8, the interaction was due mainly to a target-present/target-absent search efficiency ratio and did not reflect different patterns of performance with the target being present or absent. Error rates were low (4.0\% of the trials) and showed no trend toward a speed-accuracy trade-off.

Pairwise comparisons were performed on the combined target-present/target-absent data and on targetpresent data alone, for each planned pair of conditions (AC, BD, DE, and CF). The results showed that search was more efficient for a consistent-polarity attachment group than for an opposite-polarity attachment group [combined, $F(1,11)=10.96, p<.01$; present only, $F(1,11)=8.09, p<.02]$, search was more efficient for a consistent-polarity contour group than for an oppositepolarity contour group [combined, $F(1,11)=15.95, p<$ .01 ; present only, $F(1,11)=15.94, p<.01]$, search was no more efficient for an opposite-polarity contour group than for a misaligned opposite-polarity group [combined, $F(1,11)=3.05, p=.11$; present only, $F(1,11)=2.82$, $p=.12]$, and search for an opposite-polarity attachment group was more efficient among individual ML wing distractors than among contour group distractors [combined, $F(1,11)=30.02, p<.001$; present only, $F(1,11)=10.99$, $p<.01]$.

\section{Discussion}

In the comparison between Conditions $\mathrm{A}$ and $\mathrm{C}$, search was more efficient when the ML wings had consistent contrast polarity $(10.0 \mathrm{msec} / \mathrm{item})$ than when they had opposite contrast polarity $(16.8 \mathrm{msec} /$ item $)$. This result suggests that grouping by attachment is sensitive to the contrast sign of the input elements, and contrasts with the conclusions in Rensink and Enns (1995), in which it was 
Target-Present Trials

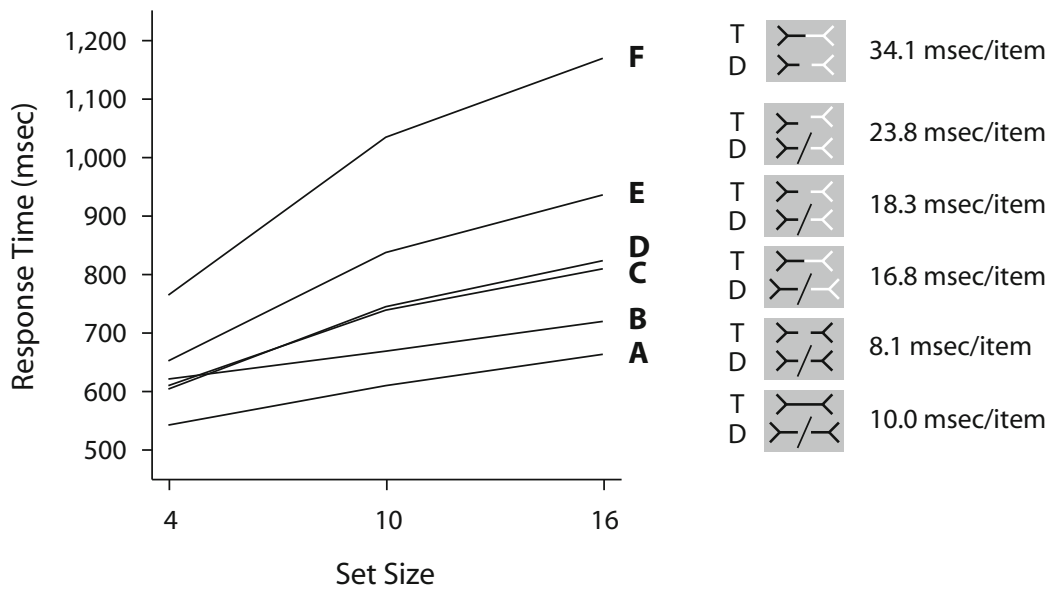

Target-Absent Trials

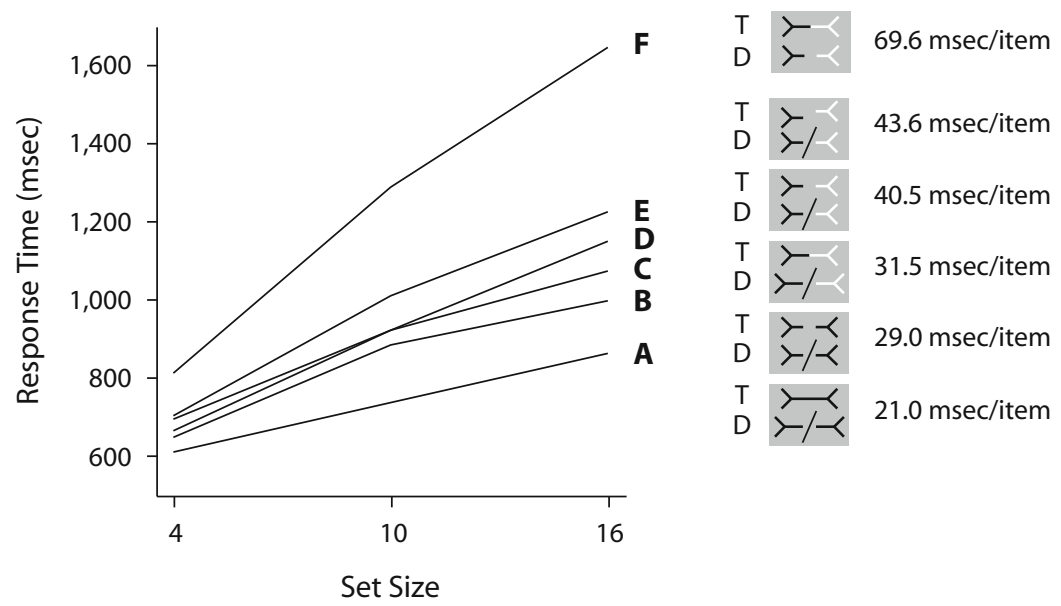

Figure 8. Results of Experiment 3. Search was more efficient when contrast polarity was consistent within an attachment group (A) and a contour group (B) than when it was reversing within a group (C and D). Search for an opposite-polarity misaligned group was fairly inefficient (E), and search for an opposite-polarity attachment group among contour groups was highly inefficient (F). Please refer to the text for more details. T, target; $D$, distractor.

suggested that connected regions are robustly grouped and support highly efficient visual search regardless of their contrast polarity. Our finding also deviates from that of Spehar's (2002) study. This discrepancy will be discussed in the General Discussion section.

The comparison between Conditions B and D revealed more efficient search for a contour group with consistent contrast polarity $(8.1 \mathrm{msec} /$ item $)$ than for one with reversing contrast polarity ( $18.3 \mathrm{msec} /$ item $)$. This result suggests that grouping by contour alignment is also sensitive to the contrast sign of the input elements. In addition, a comparison between Conditions D and E revealed that an oppositepolarity contour group supported no more efficient search (18.3 msec/item) than did an opposite-polarity misaligned group (23.8 msec/item). This result suggests that contour alignment was quite limited in facilitating grouping when it was acting on reversing contrast inputs, and was in contrast with the results for Conditions $\mathrm{B}$ and $\mathrm{C}$ in Experiment 2 , in which a significant contribution from contour alignment in facilitating grouping was measured.

Our conclusions deviate from those in Rensink and Enns's (1995) study, which showed no effect from reversing contrast polarity on the robustness and search efficiency of short-range contour grouping (Experiment 4A, consistent polarity, $3.1 \mathrm{msec} /$ item; Experiment $5 \mathrm{C}$, opposite polarity, $4.0 \mathrm{msec} / \mathrm{item})$. Note that even though we replicated their opposite-polarity results in terms of search efficiency in Experiment 1 (3.6 msec/item), search with an identical target in this experiment resulted in a much less efficient search (18.3 msec/item). Since the same target was employed, it was unlikely that the discrepancy between this experiment and our replication of Rensink 
and Enns's result was due to a difference in grouping processes. As we discussed previously, we reason that this discrepancy was due to search being at least partially supported by individual junction shapes in Rensink and Enns's experiments and in our Experiment 1.

The comparison between Conditions $\mathrm{C}$ and $\mathrm{F}$ revealed more efficient search for an opposite-polarity attachment group when the distractors were unpaired individual ML wings $(16.8 \mathrm{msec} /$ item $)$ than when the distractors were paired (34.1 msec/item). As was reasoned above, if no grouping of distractors occurred in Condition F, this condition would be functionally identical to Condition A, so one would expect them to produce similar search efficiencies. The present result therefore suggests that some kinds of grouping still occurred across the opposite-polarity pairs of ML wings. However, since we also found in Condition A that opposite-polarity attachment grouping itself was not very efficient, it was possible that the participants engaged in a strategy to check each item for a complement. In this case, the present result would reflect that this strategy was rendered less plausible when each distractor was a pair of ML wings (so that each always had a complement) than when the distractors were individual ML wings (without a complement).

Taken together, the results of Experiments 2 and 3 constituted the main findings of the present study. We found that contour grouping requires some amount of attentional resources in order to operate and that it occurs only for items with consistent contrast polarity. The contribution from contour alignment appeared to be quite limited when perceptual grouping occurred across reversing contrast polarity. We also found that attachment grouping is sensitive to contrast polarity.

There were three potential issues to be addressed before we could accept these conclusions. The first issue concerned the generality of the results. Experiments 4 and 5 were conducted to address this concern. The second issue was about a potential confound between a sensitivity to contrast polarity and an effect from distractor heterogeneity. Experiment 6 was conducted to address this confound. The third issue was related to the failure in Experiment 3 to replicate the efficiency difference between searches supported by attachment and contour groups that was previously observed in Experiment 2. Experiment 7 addressed this issue.

\section{EXPERIMENT 4}

In our previous experiments, we examined attachment grouping and contour grouping by using only Y-shaped ML wings. The use of a single kind of stimulus may place a limit on the generality of the conclusions that we could reach. This is especially true since ML configurations composed of Y-shaped ML wings may produce a lower degree of perceptual closure, as compared with other stimuli, such as those composed of arrow-shaped ML wings. Perceptual closure refers to the extent to which a figure is perceived as a closed region, as opposed to a collection of line segments. It is possible that insensitivity to contrast polarity in perceptual grouping is specific to stimuli with a high degree of perceptual closure. For instance, the reverse-contrast Kanizsa figure (Figure 1), the shape of the square groups in Gilchrist et al.'s (1997) study, and the shapes used in Spehar's (2002) study (see Figure 14) were all readily perceived as closed shapes.

In Experiment 4, we verified our results by using a set of stimuli - arrow-shaped ML wings - that produced a higher degree of perceptual closure. If we were able to attain a pattern of performance that was similar to that in Experiment 3 , our conclusions could be generalized to a wider set of stimuli with different degrees of perceptual closure.

\section{Method}

Participants. Twelve students from the University of Hong Kong participated in this experiment. All had normal or corrected-tonormal vision. They were paid HK $\$ 40$ for a 40 -min session.

Apparatus and Stimuli. All aspects of this experiment were identical to those in Experiment 3, except that all Y-shaped ML wings were replaced by arrow-shaped ML wings. The arrow-shaped ML wing stimuli were modified from the arrow stimuli used in Experiment 1 according to identical specifications for Experiment 3.

Design. The design of this experiment was identical to that in Experiment 3.

Procedure. The procedure was identical to that in Experiment 2.

\section{Results}

Data were screened and search efficiency was obtained as in Experiment 1. The results are shown in Figure 9. A two-way repeated measures ANOVA was performed, with search efficiency as the dependent variable and target presence and test condition as independent variables. The results showed that search was more efficient for targetpresent trials $[F(1,11)=31.58, p<.001]$ and search efficiency differed across test conditions $[F(5,55)=24.57$, $p<.001]$. The interaction between target presence and test condition was not significant $[F(5,55)=1.57, p>.1]$. Error rates were low (3.7\% of the trials) and showed no trend toward a speed-accuracy trade-off.

As in Experiment 3, pairwise comparisons were performed on the combined target-present/target-absent data and on the target-present data alone for each planned pair of conditions (AC, BD, DE, and $\mathrm{CF}$ ). The results were consistent with those in Experiment 3. First, search for an attachment group was more efficient when it had consistent-polarity than when it had opposite-polarity wings [combined, $F(1,11)=8.10, p<.02$; present only, $F(1,11)=26.08, p<.001]$. Similarly, search for a contour group was more efficient when it was consistent in its contrast polarity [combined, $F(1,11)=15.41, p<.01$; present only, $F(1,11)=31.24, p<.001]$. Search was no more efficient for an opposite-polarity contour group than for a misaligned opposite-polarity group [combined, $F(1,11)=$ $2.20, p>.1$; present only, $F(1,11)=0.22, p>$.6]. Finally, search for an opposite-polarity attachment group was more efficient among individual ML wing distractors than among paired ML wing distractors [combined, $F(1,11)=35.08, p<.001$; present only, $F(1,11)=73.96$, $p<.001]$.

\section{Discussion}

The results are consistent with those in Experiment 3. In the comparison between Conditions $\mathrm{A}$ and $\mathrm{C}$, search 
Target-Present Trials

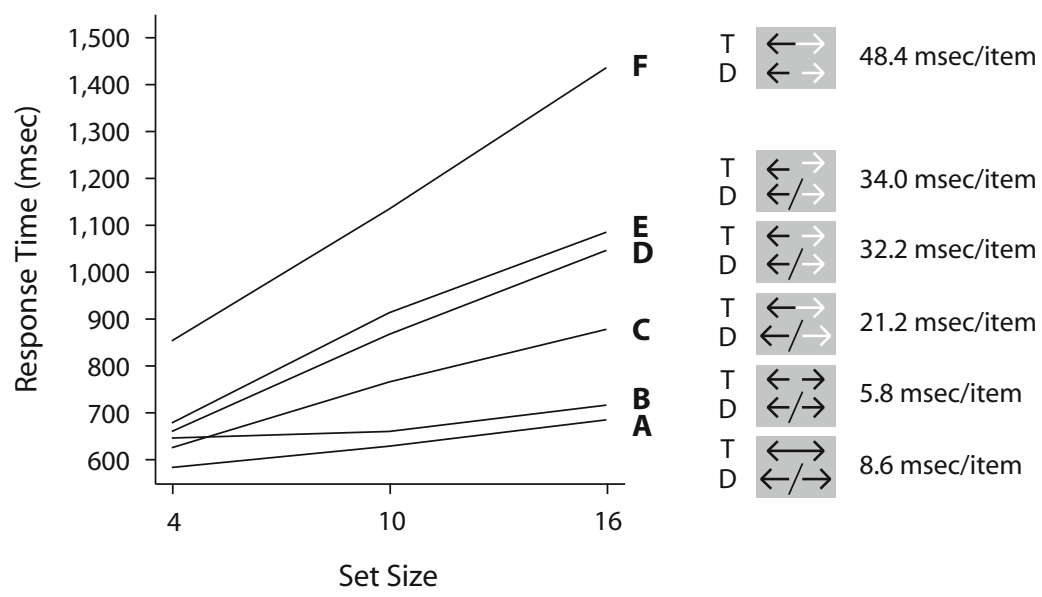

Target-Absent Trials

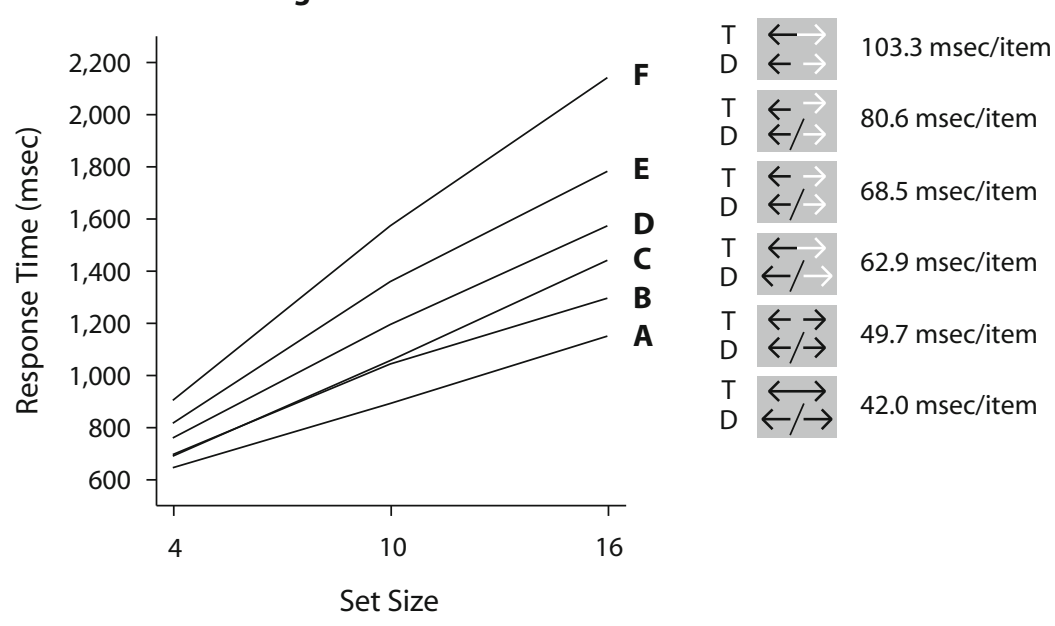

Figure 9. Results of Experiment 4. By using arrow-shaped stimuli, the results basically replicated those of Experiment 3. T, target; D, distractor.

was more efficient for a consistent-polarity $(8.6 \mathrm{msec} /$ item) than for an opposite-polarity $(21.2 \mathrm{msec} /$ item $)$ attachment group. This confirms our conclusion that attachment grouping is sensitive to changes in contrast polarity. The comparison between Conditions B and D showed that search was more efficient for a consistent-polarity (5.8 msec/item) than for an opposite-polarity $(32.2 \mathrm{msec} /$ item) contour group. This confirms our conclusion that contour grouping is also sensitive to contrast polarity. The search efficiency difference between Conditions D and E was not significant, showing similar search efficiency when opposite-polarity contour grouping was aligned (32.2 msec/item) and was misaligned $(34.0 \mathrm{msec} /$ item). This suggests that when the constituent parts of a perceptual group have different contrast polarity, contour alignment does not facilitate the grouping process, and is consistent with a strong form of the hypothesis that contour grouping is sensitive to contrast polarity. Finally, the comparison between Conditions $\mathrm{C}$ and $\mathrm{F}$ revealed more efficient search for an opposite-polarity attachment group among individual ML wings than among paired ML wings. This result suggests that some grouping might have occurred across the opposite-polarity paired ML wings or that a complement-checking strategy was rendered inefficient by the paired ML wing distractors.

\section{EXPERIMENT 5}

The consistency between Experiments 3 and 4 suggests that our conclusions are applicable to grouping with different degrees of perceptual closure. On the other hand, the stimuli used in Experiments 3 and 4 were common, in the sense that both were composed of several line segments. To further generalize our findings, in Experiment 5, we created stimuli that also produced a strong closure perception but were composed of filled 2-D shapes. Therefore, results similar to those in the previous experiments with the use of these stimuli would ensure that our conclusions were not specific to a particular degree of perceptual closure, or even to a particular kind of stimulus. 
A

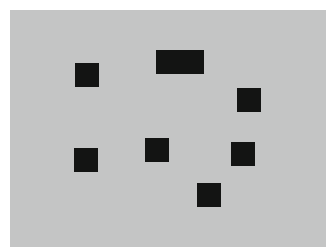

C

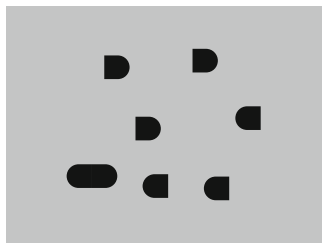

B

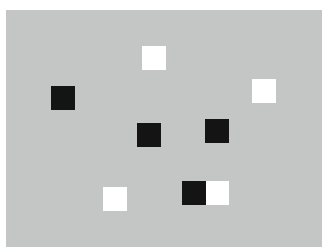

D

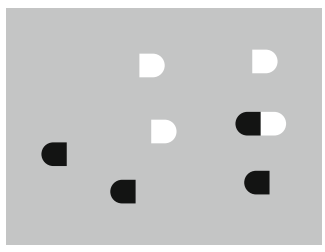

Figure 10. Illustration of search displays in Experiment 5. (A) Search for a consistent-polarity attachment group among individual squares. (B) Search for an opposite-polarity attachment group among individual squares. (C) Search for a consistentpolarity attachment group among individual shapes. (D) Search for an opposite-polarity attachment group among individual shapes.

The testing conditions are illustrated in Figure 10. In Conditions A and B, we asked the participants to search for a horizontal rectangle among squares, in which each rectangle was the size of two distractor squares. The target was a combination of two consistent-polarity squares in Condition A and was a combination of reversing polarity squares in Condition B. In Conditions C and D, the target was a round-ended rectangular shape. The distractors were split halves of this shape. In Condition C, the target had a consistent contrast polarity; in Condition D, the target was composed of opposite-polarity halves.

\section{Method}

Participants. Eight students from the University of Hong Kong participated in this experiment. All had normal or corrected-tonormal vision. They were paid HK\$30 for a 30-min session.

Apparatus and Stimuli. The apparatus involved and the setting of this experiment were identical to those specified in Experiment 2, except for the following. Set sizes of 4, 8, and 12 were used, and in this experiment, the set sizes were defined by the number of items, instead of the number of ML wings. The items were assigned to invisible cells of a $6 \times 4$ table of dimensions $16.8^{\circ} \times 11.2^{\circ}$. For each set size, the appropriate number of cells was chosen randomly from the 24 cells, and each chosen cell was assigned a search item. The closest possible distance between the center of any item and any cell border was $0.92^{\circ}$. With regard to the stimuli, in Conditions A and B, the target was a $2^{\circ} \times 1^{\circ}$ rectangle, and the distractors were $1^{\circ} \times 1^{\circ}$ squares. In Conditions $\mathrm{C}$ and $\mathrm{D}$, the target was a $2^{\circ} \times 1^{\circ}$ round-ended rectangle (which was equivalent to connecting a $1^{\circ} \times 1^{\circ}$ square between the left and right halves of a circle with a $0.5^{\circ}$ radius), and the distractors were left and right halves of the target. For Conditions A and $\mathrm{C}$, the target and the distractors had consistent contrast polarity within a trial; the contrast polarity was chosen randomly for each trial. For Conditions B and D, the left and right halves of the target had opposite contrast polarity, and the distractors were assigned a contrast polarity according to their facing direction with reference to the target of the same trial; contrast polarity of each side of the target was chosen randomly for each trial.

Design. The design of this experiment was identical to that in Experiment 2.

Procedure. The procedure was identical to that in Experiment 2.

\section{Results}

The data were screened and search efficiency was obtained as in Experiment 1. The results are shown in Figure 11. A two-way repeated measures ANOVA was performed, with search efficiency as the dependent variable and target presence and test condition as independent variables. The results showed that search efficiency differed across test conditions $[F(3,21)=16.89, p<.001]$, but search was not more efficient for target-present trials than for target-absent trials $[F(1,7)=1.96, p>.2]$. The interaction between target presence and test condition was not significant $[F(3,21)=0.07, p>.9]$. Error rates were low (1.7\% of the trials) and showed no trend toward a speedaccuracy trade-off.

Pairwise comparisons were performed on the combined target-present/target-absent data and on target-present data alone, for each planned pair of conditions (AB and CD). In both comparisons, search was more efficient with consistent-polarity stimuli $[\mathrm{AB}$, combined, $F(1,7)=$ $20.50, p<.01$; present only, $F(1,7)=5.19, p=.06$; $\mathrm{CD}$, combined, $F(1,7)=34.22, p<.001$; present only, $F(1,7)=75.43, p<.001]$.

\section{Discussion}

The results are consistent with the findings in Experiment 3. With both kinds of stimuli, attachment grouping supported more efficient visual search when its constituents had consistent contrast polarity than when its constituents had reversing contrast polarity (squares, 6.2 vs. $13.5 \mathrm{msec} /$ item; round shapes, $3.3 \mathrm{vs}$. $12.2 \mathrm{msec} / \mathrm{item}$ ). These results suggest that our previous conclusions are not limited to ML stimuli, or even to line segments in general, and are general to filled-shape stimuli. Attachment grouping is shown to be sensitive to the contrast sign of its inputs. This conclusion is consistent with Palmer and Rock's (1994) suggestion that the entry-level element for grouping is defined by uniform connectedness. Nonuniform connectedness, such as connected shapes with reversing contrast polarity, seems to support less efficient grouping than does uniform connectedness.

\section{EXPERIMENT 6}

In Experiment 6, we addressed an issue with Conditions A-D in Experiments 3, 4, and 5, in which our contrast polarity manipulation was confounded with the distractor heterogeneity conditions. For instance, in these conditions, when the target had a consistent contrast polarity, the distractors were homogeneous in terms of contrast polarity; whenever the target had reversing contrast polarity, the distractors became heterogeneous in terms of contrast polarity. Therefore, it was possible that the observed sensitivity to contrast polarity in these conditions may actually reflect an effect of distractor heterogeneity, which can reduce search efficiency (e.g., Duncan \& Humphreys, 1989). We addressed this confound by replicating Conditions A-D of Experiment 3, while using reversing contrast polarity distractors in all the conditions and manipulating only the contrast po- 
Target-Present Trials

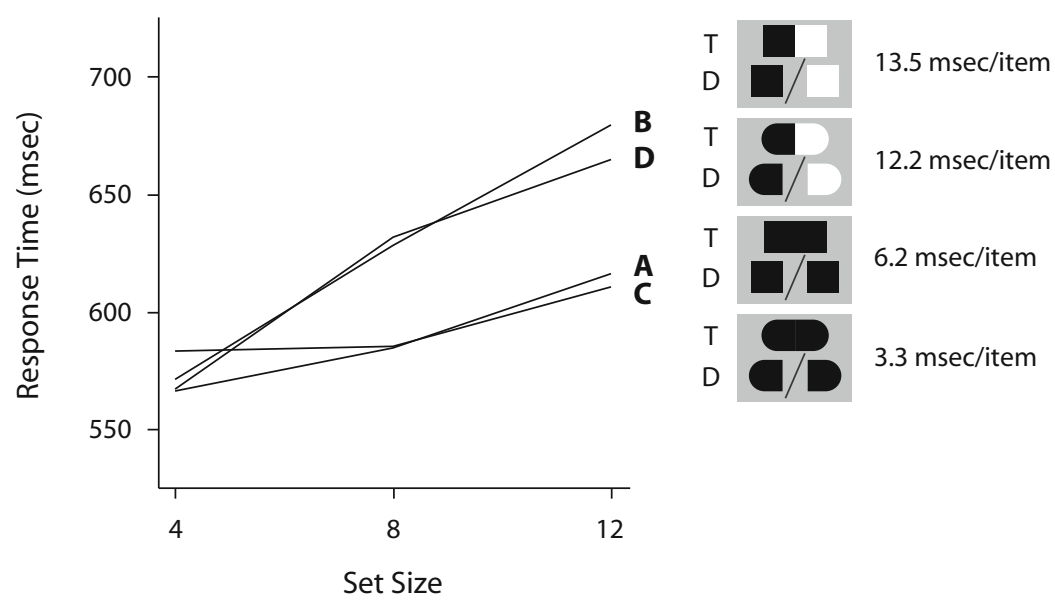

Target-Absent Trials

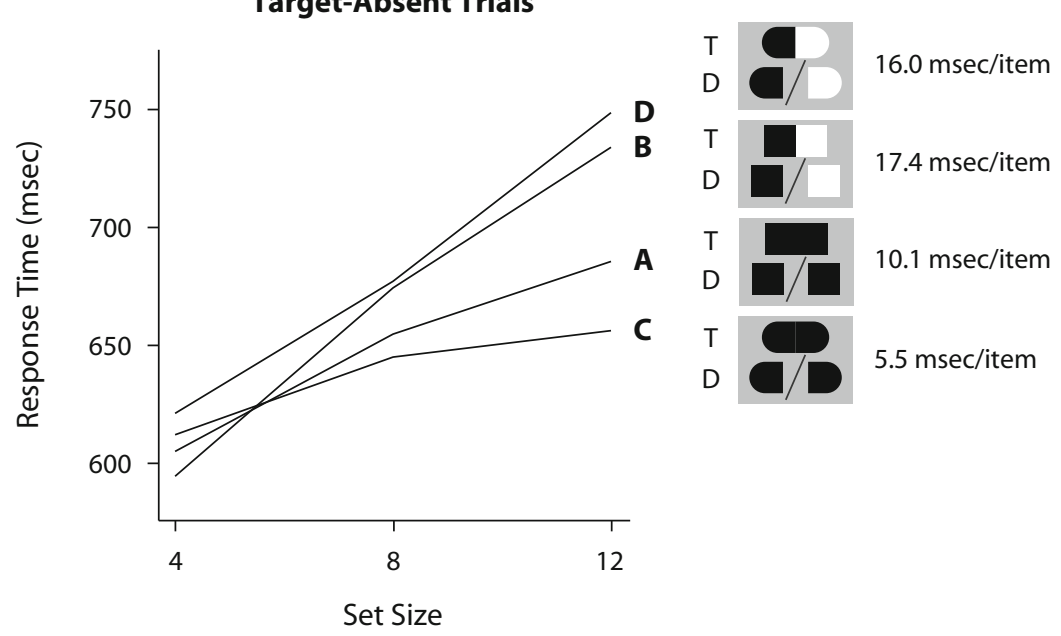

Figure 11. Results of Experiment 5. Search was more efficient when contrast polarity was consistent within an attachment group ( $A$ and $C$ ) than when it was reversing within a group (B and $D)$. T, target; $D$, distractor.

larity of the targets. This way, any difference between consistent-polarity and opposite-polarity conditions must be due to a sensitivity to contrast polarity of the grouping process that acted on the target and cannot be due to the distractors' being heterogeneous in some but not other conditions. If attachment grouping and contour grouping were truly sensitive to contrast polarity, search would be more efficient in Condition A than in Condition $\mathrm{C}$ and in Condition $\mathrm{B}$ than in Condition D.

\section{Method}

Participants. Eight students from the University of Hong Kong participated in this experiment. All had normal or corrected-tonormal vision. They were paid HK $\$ 20$ for a 20 -min session.

Apparatus and Stimuli. All aspects of this experiment were identical to those in Conditions A-D of Experiment 3, except that the contrast polarity of the distractors varied within each search display in all the conditions. As a note, the targets in Conditions A and $\mathrm{C}$ had a consistent contrast polarity within a trial, and varied randomly between trials; the targets in Conditions B and D had reversing contrast polarity within a trial.
Design. The design of this experiment was identical to that in Experiment 2.

Procedure. The procedure was identical to that in Experiment 2.

\section{Results}

The data were screened and search efficiency was obtained as in Experiment 1. The results are shown in Figure 12. A two-way repeated measures ANOVA was performed, with search efficiency as the dependent variable and target presence and test condition as independent variables. The results showed that search was more efficient for target-present trials $[F(1,7)=48.70, p<.001]$ and search efficiency differed across test conditions $[F(3,21)=17.84$, $p<.001]$. The interaction between target presence and test condition was not significant $[F(3,21)=0.86, p>4]$. Error rates were low (1.8\% of the trials) and showed no trend toward a speed-accuracy trade-off.

Pairwise comparisons were performed on the combined target-present/target-absent data and on target-present data alone, for each planned pair of conditions (AC and $\mathrm{BD}$ ). 
Target-Present Trials

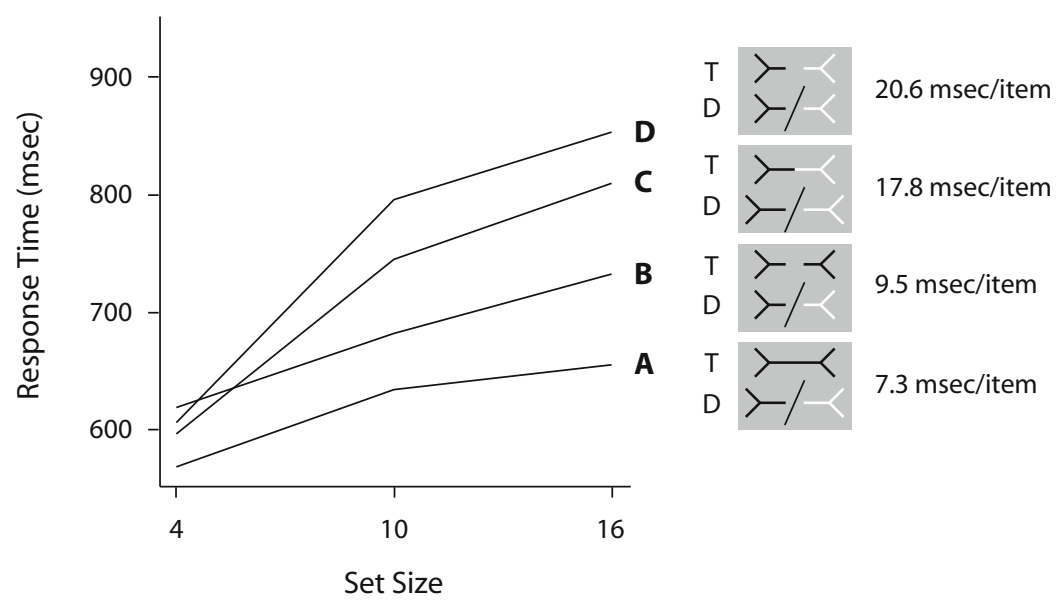

Target-Absent Trials

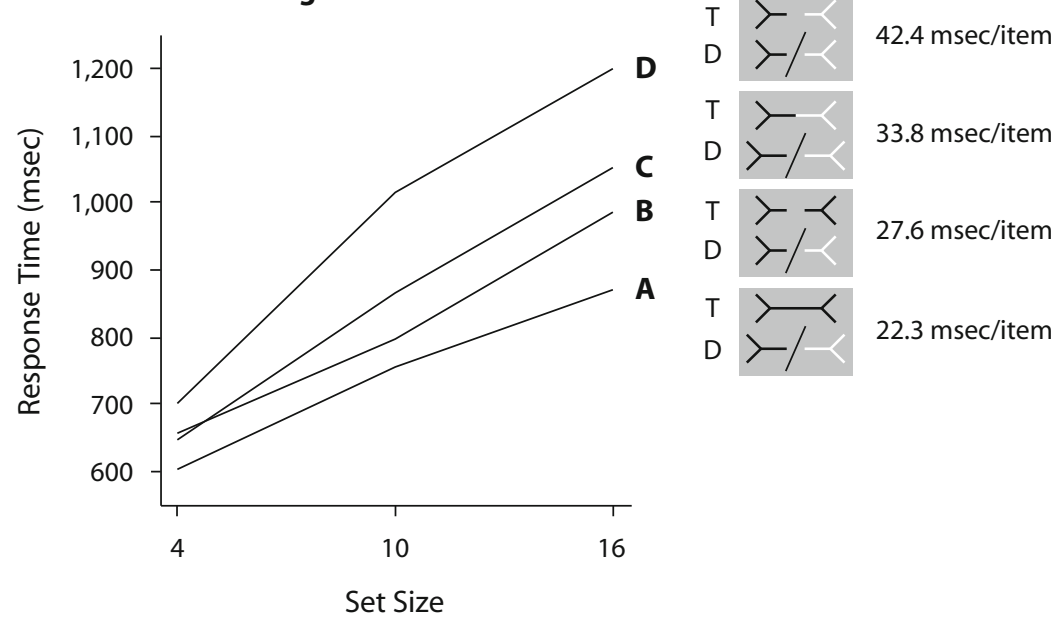

Figure 12. Results of Experiment 6. The contrast polarity of the distractors was reversing in all the conditions of this experiment. The results basically replicated those of Experiment 3. Search was more efficient when contrast polarity was consistent (randomly either both black or both white) within an attachment target (A) and a contour target (B) than when it was reversing within a group (C and D). T, target; D, distractor.

Consistent with our findings in Experiment 3, the results showed more efficient search for a consistent-polarity attachment group than for an opposite-polarity attachment group [combined, $F(1,7)=15.02, p<.01$; present only, $F(1,7)=32.86, p<.001]$. Similarly, search was more efficient for a consistent-polarity contour group than for an opposite-polarity contour group [combined, $F(1,7)=$ $25.71, p<.01$; present only, $F(1,7)=10.79, p<.02]$.

\section{Discussion}

The results are consistent with the results of Experiments 3, 4, and 5. In the comparison between Conditions $\mathrm{A}$ and $\mathrm{C}$, search was more efficient for a consistentpolarity attachment group ( $7.3 \mathrm{msec} /$ item $)$ than for an opposite-polarity attachment group (17.8 msec/item). This confirms our finding that attachment grouping is sensitive to contrast polarity. The comparison between Conditions B and D showed more efficient search for contour grouping with consistent $(9.5 \mathrm{msec} / \mathrm{item})$ than with reversing (20.6 msec/item) contrast polarity ML wings. This confirms our finding that contour grouping depends on input from the same contrast sign. It is also noteworthy that the magnitude of the search efficiency in this experiment is comparable to that of each corresponding condition in Experiment 3, showing that distractor heterogeneity in terms of contrast polarity did not affect search in general, and suggests that the results of Experiment 3 are generally valid. We reason that the lack of an effect from contrast polarity heterogeneity was due to visual search in our experiment being based on the length dimension. It has been shown previously that distractor heterogeneity in a task-irrelevant perceptual dimension 
generally does not reduce visual search efficiency (e.g., Treisman, 1988).

\section{EXPERIMENT 7}

Experiment 7 addressed the finding that the efficiency difference observed in Experiment 2 between searches supported by attachment grouping and contour grouping was not replicated in Conditions A and B of Experiments 3, 4, and 6. In Experiment 2, the lower search efficiency of contour grouping (Condition $\mathrm{B}$, as compared with Condition A, in which search was supported by attachment grouping) is taken to argue that contour grouping requires more attentional resources to operate than are needed for attachment grouping. This is a major conclusion of the present study. However, the failure to replicate this result in three later experiments poses an important threat to the soundness of our conclusion. The result of Experiment 2 itself also needs to be considered carefully, since the efficiency difference between its Conditions A and B was only marginally significant $(p=.06)$ with target-present responses. Therefore, in this experiment, we attempted to verify the findings of Experiment 2 by replicating Conditions A and B with both a normal and a longer set of stimuli.

It should be noted that the failure to replicate the result of Experiment 2 in Experiments 3, 4, and 6 was not without reason. Specifically, longer distractor wings $\left(1.1^{\circ}\right)$ were used with the attachment target but shorter distractor wings $\left(0.82^{\circ}\right)$ were used with the contour target in these experiments, in which both targets had an identical length. Since search was based on identifying length differences, longer distractor wings would render search less efficient, putting the search for an attachment group in an unfair condition. Therefore, even if attachment grouping better facilitated perceptual grouping in these experiments, this advantage might have been offset by the use of longer distractor wings and might not have been reflected in terms of visual search efficiency. In the present experiment, we always used normal-length distractor wings $\left(0.82^{\circ}\right)$ and targets $\left(2.1^{\circ}\right)$ in Conditions $\mathrm{A}$ and $\mathrm{B}$ and longer distractor wings $\left(1.1^{\circ}\right)$ and targets $\left(2.6^{\circ}\right)$ in Conditions $\mathrm{C}$ and D. By equating the target-distractor length ratios in comparisons of attachment and contour groups, this experiment would allow a fairer test for the extent to which attachment and contour grouping require spatial attention.

\section{Method}

Participants. Twelve students from the University of Hong Kong participated in this experiment. All had normal or corrected-tonormal vision. They were paid HK $\$ 20$ for a 20 -min session.

Apparatus and Stimuli. The apparatus involved and the setting of this experiment were identical to those specified in Experiment 2, and the stimuli were as specified in the following. In Conditions A and $\mathrm{C}$, the targets were attached ML configurations; in Conditions B and $\mathrm{D}$, the targets were paired ML wings. In all the conditions, the distractors were left- and right-facing individual ML wings. In Conditions $\mathrm{A}$ and $\mathrm{B}$, a full $\mathrm{ML}$ target was $2.1^{\circ}$ long, the gap size for a contour group was $0.47^{\circ}$, and the distractor wings were $0.82^{\circ}$ long. These settings were the same as those in Experiment 2. For Conditions $\mathrm{C}$ and $\mathrm{D}$, a full ML target was $2.6^{\circ}$ long, the gap size for a contour group was $0.47^{\circ}$, and the distractor wings were $1.1^{\circ}$ long. This distractor length was identical to that in Condition A of Experiments 3,4 , and 6 .

Design. The design of this experiment was identical to that in Experiment 2.

Procedure. The procedure was identical to that in Experiment 2.

\section{Results}

The data were screened and search efficiency was obtained as in Experiment 1. The results are shown in Figure 13. A two-way repeated measures ANOVA was performed, with search efficiency as the dependent variable and target presence and test condition as independent variables. The results showed that search was more efficient for target-present trials $[F(1,11)=15.73, p<.01]$ and search efficiency differed across test conditions $[F(3,33)=14.02$, $p<.001]$. The interaction between target presence and test condition was not significant $[F(3,33)=2.23, p=.10]$. Error rates were low (2.7\% of the trials) and showed no trend toward a speed-accuracy trade-off.

Pairwise comparisons were performed on the combined target-present/target-absent data and on target-present data alone, for each planned pair of conditions ( $\mathrm{AB}$ and $\mathrm{CD}$ ). The results showed more efficient search for an attachment group than for a contour group with both normallength stimuli [combined, $F(1,11)=31.78, p<.001$; present only, $F(1,11)=13.79, p<.01]$ and longer length stimuli [combined, $F(1,11)=24.33, p<.001$; present only, $F(1,11)=7.80, p<.02]$. These results confirm the findings of Experiment 2.

\section{Discussion}

Using either a normal- or a longer length stimulus, we found that search was more efficient for attachment groups (Condition A, $2.9 \mathrm{msec} / \mathrm{item}$; Condition C, $4.4 \mathrm{msec} /$ item) than for contour groups (Condition B, $7.9 \mathrm{msec} /$ item; Condition D, $9.0 \mathrm{msec} /$ item). These results were comparable to those obtained in Experiment 2 in terms of their magnitude (Experiment 2A, $3.8 \mathrm{msec} / \mathrm{item}$; Experiment $2 \mathrm{~B}, 9.2 \mathrm{msec} / \mathrm{item})$. These results confirm our previous conclusion that contour grouping requires more attention to operate than does attachment grouping and provide evidence that our previous failure to replicate this result was due to confounding differences in stimulus length between the two conditions.

\section{GENERAL DISCUSSION}

In the present study, we tested two traditional assumptions of perceptual grouping by contour alignment: that (1) it occurs at a relatively low level of visual processing, and (2) it occurs across changes in contrast polarity (e.g., Grossberg \& Mingolla, 1985; Kellman \& Shipley, 1991). We also tested the assumption that grouping by direct attachment occurs across reversing contrast polarity, at least when contrast polarity does not reverse at junction points of line segments (e.g., Rensink \& Enns, 1995; Spehar, 2002). Contrary to these expectations, we showed in seven experiments that grouping by contour alignment required some amount of attention to oper- 
Target-Present Trials

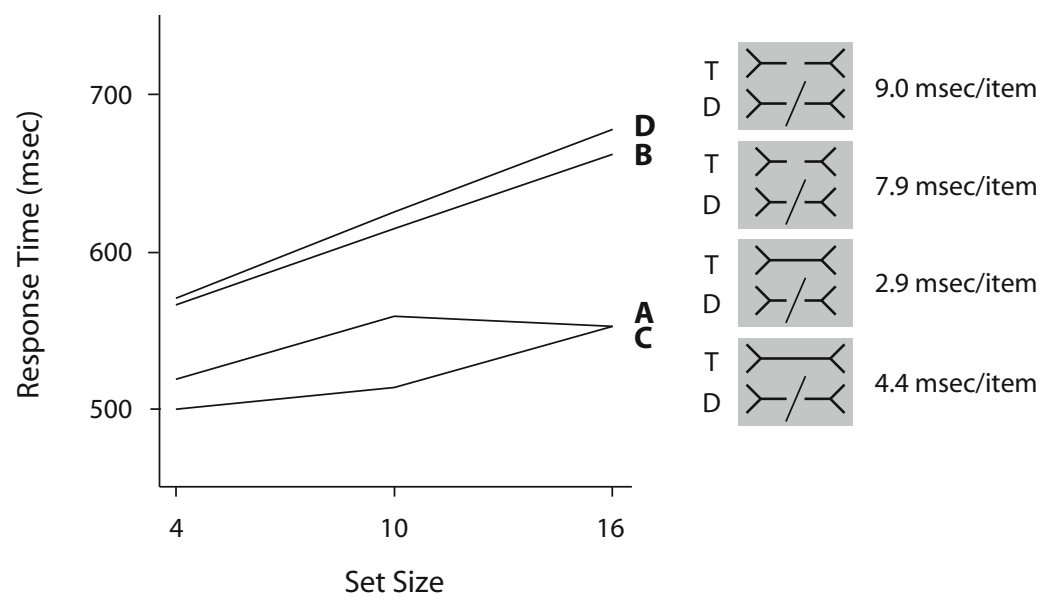

Target-Absent Trials

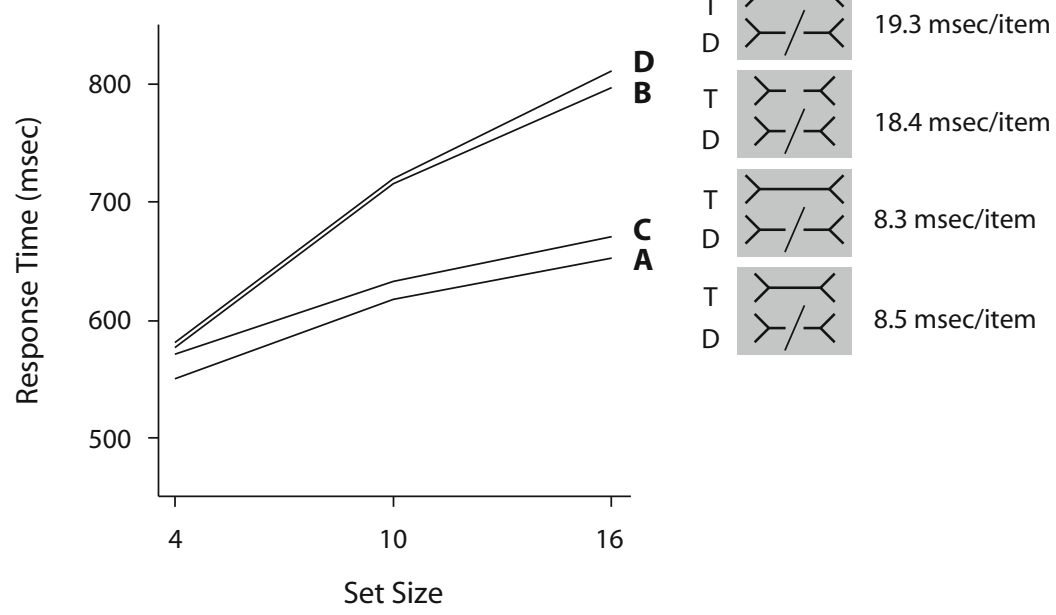

Figure 13. Results of Experiment 7. The results in this experiment replicated those of Experiment 2. With both normal and longer Müller-Lyer stimuli, search was more efficient for an attachment group (A and C) than for a contour group (B and D). $T$, target; D, distractor.

ate, so that only grouping by attachment might be truly regarded as a preattentive process (Experiments 2 and 7). Perceptual grouping by either contour alignment or attachment was shown to be sensitive to reversals of the contrast signs of its constituent elements (Experiments 3, 4, 5, and 6). When comparing between aligned and misaligned groups of separated elements, we showed that contour alignment facilitated perceptual grouping when it occurred for consistent contrast polarity elements (Experiment 2), but not when it occurred across reversing contrast polarity (Experiments 3 and 4). These results suggest that contour grouping relies on consistent contrast polarity input to operate. ${ }^{3}$ We showed that our findings were not specific to Y-shaped ML stimuli (Experiments 4 and 5) and were not due to a confound with distractor heterogeneity (Experiment 6). Taken together, the present findings call into question some common assumptions of theories of perceptual grouping and cast doubt on the generality of some previous findings that supported these assumptions.

Why is there a discrepancy between previous findings and the present results? Several reasons may be important. The first concerns the preattentive status of contour grouping. Although it was suggested previously that contour grouping occurs preattentively (Gilchrist et al., 1997; Rensink \& Enns, 1995), our findings suggest that contour grouping is a process that requires spatial attention in order to operate. Of course, this discrepancy stems at least partially from different views of the criterion for a fully parallel search. For example, Gilchrist et al. found that contour grouping supported a search efficiency of $12.4 \mathrm{msec} /$ item. This fairly efficient performance was regarded as a benchmark for preattentive processing, in their view, and was taken to suggest that contour grouping occurred preattentively. However, in our experiments, the same level of efficiency was interpreted as requiring atten- 
tion. Obviously, it is not the aim of this study to resolve the enduring issue of the visual search efficiency that should be regarded as marking fully parallel search. On the other hand, we maintain the more important proposal that the extent to which contour grouping requires spatial attention contrasts with and is empirically distinguishable from that of attachment grouping. Attachment grouping appeared not to require spatial attention in order to operate in our study and in previous reports. This position is consistent with the conjecture that uniformly connected regions constitute the entry-level unit for perceptual grouping (Palmer \& Rock, 1994). In this case, whereas grouping would not be required for attached elements (with consistent surface properties), some attentive grouping processes may be required for separated elements. Therefore, our conclusion is that attachment grouping and contour grouping are operating with different efficiencies and should not be collectively regarded as being preattentive.

The second discrepancy lies in the sensitivity to contrast polarity of attachment and contour grouping. On the one hand, we have demonstrated that grouping efficiency was reduced by contrast polarity reversals on a number of occasions. On the other hand, it is difficult to deny that, sometimes, perceptual grouping manifests itself as being polarity insensitive. For instance, Rensink and Enns (1995) and Spehar (2002) showed concrete evidence that grouping supported by line segments of opposite contrast polarity and that supported by consistent contrast polarity line segments were equally efficient. Their stimuli are illustrated in Figure 14. In Rensink and Enns's Experiment $1 \mathrm{~A}$ and in Spehar's Experimental Condition B (Figures 14A and 14D), visual search supported by attachment grouping was highly efficient. In Rensink and Enns's Experiment 4D and in Spehar's Control Conditions B and D (Figures 14C, 14F, and 14H), search was generally inefficient when some line segments connecting the rest of a group were removed, resulting in a proximity-based group. An important condition was that when they reconnected the broken group with line segments of an opposite contrast polarity (Rensink \& Enns's Experiment 5A and Spehar's Control Conditions A and C; Figures 14B, $14 \mathrm{E}$, and 14G), search was as efficient as in their attachment grouping conditions. This result shows that the opposite-polarity line segments contributed to grouping and supported highly efficient search. In these situations, grouping across changes in contrast polarity was evidently performed as efficiently as attachment grouping.

One observable difference between the stimulus designs in Rensink and Enns's (1995) and Spehar's (2002) studies and those in the present study is that whereas, in their studies, proximity-based grouping would still occur without the connecting opposite-polarity line segments, in our study, no grouping would occur in this case. That is, in their opposite-polarity conditions (Figures 14B, 14E, and $14 \mathrm{G}$ ), the target could still be differentiated from the distractors without the line elements of one of the contrast signs (as in Figures 14C, 14F, and 14H). However, in our study, target identification would not be possible without considering inputs of both contrast signs. If it was

\section{Search \\ Target Distractor Efficiency}

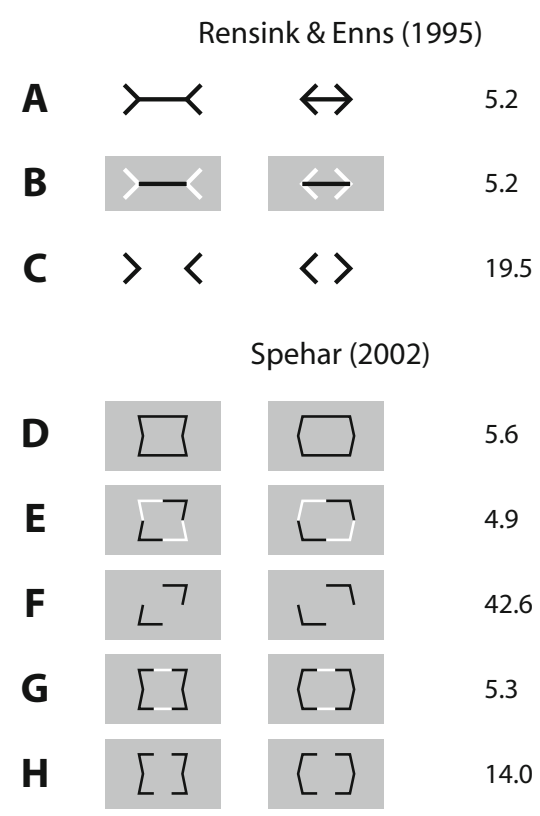

Figure 14. Stimuli and results from Rensink and Enns (1995) and Spehar (2002). Although we found in our experiments that efficiency of attachment grouping was reduced by reversing its contrast polarity, this result was not observed in their studies. Panels A-H correspond to Rensink and Enns's Experiments 1A, 5A, and 4D, Spehar's Experimental Condition B, and Spehar's Control Conditions A-D, respectively. Search efficiency refers only to target-present trials, in milliseconds per item.

this difference that underlies the different results between our study and these earlier ones, it is possible that elements of one contrast sign in their stimuli may serve only to strengthen the preexisting groups of the other contrast sign, and thus highly efficient visual search might be supported; this high efficiency may not be attributed to the otherwise less efficient grouping between elements of both contrast signs. On the other hand, this mechanism was inapplicable for the opposite-polarity conditions in our experiments, because there was no possibility of identifying the target by considering only inputs of one contrast sign. Therefore, search remained inefficient because it must rely on grouping of elements of differing contrast polarity, a process that is relatively inefficient. This explanation is, of course, highly speculative and requires further investigation.

In terms of contour grouping, as was noted above, discrepancies exist between the present study and Gilchrist et al.'s (1997) and Rensink and Enns's (1995) studies. Our results suggest that Rensink and Enns's demonstration of an insensitivity to contrast polarity in contour grouping (the short-range condition) was due to that search being at least partially facilitated by isolated junction shapes. However, the cause of the difference between Gilchrist et al. and our study is not so clear. Gilchrist et al. found that grouping of two aligned squares was not more ef- 
ficient in the consistent-polarity condition $(10.8 \mathrm{msec} /$ item) than in the opposite-polarity condition $(12.4 \mathrm{msec} /$ item). This suggests an insensitivity to contrast polarity in contour grouping. Nevertheless, since their search in the consistent-polarity condition was numerically more efficient than that in their opposite-polarity condition, it may be the case that the observed insensitivity was due to a lack of statistical power.

The conclusions that we have drawn here are not without possible challenge. For instance, in the whole study, we have assumed that visual search efficiency is a valid indicator of the extent to which grouping requires attention. It must be noted, however, that visual search efficiency may also be influenced by other factors, such as the perceived length difference between targets and distractors or the quality of the length representation of a perceptual group. ${ }^{4}$ Our preference for interpreting search efficiency as grouping efficiency is due mainly to the lack of any reason to believe that the length estimates of perceptual groups would be influenced by our manipulation. Although noisier length estimates may result from particular grouping conditions, it remains reasonable to believe that it was, in turn, due to a poor grouping representation. Since attention may serve to improve grouping representations, we regard our interpretation as remaining viable in this case.

With regard to Rensink and Enns's (1995) low-level grouping theory, we do not propose that its general validity was challenged by the possibility of junction-based search. Many of the conditions in their study did not suffer from this confound. Indeed, preemption, the sign for robust grouping, is a valid indicator of the low-level status of perceptual grouping. If attention is not able to break apart a perceptual group, it seems reasonable to suggest that such grouping occurs at a low level. For example, it is clear that junction-based search does not confound the comparison between Rensink and Enns's Experiments $6 \mathrm{~B} 2$ and $6 \mathrm{C} 2$. Their corresponding conclusion that long-range grouping occurred at a low level only with intersectable contours verifies our present findings that contour grouping is sensitive to contour alignment (Experiment 2). In the present study, we made use of search efficiency as the indicator of grouping, so that we were able to examine situations in which the method of preemption fails when junction-based search is confounded with the critical comparisons.

An overview of the present results suggests that our data are consistent with a grouping mechanism that employs simple spatial filtering. ${ }^{5}$ According to this view, search was generally rapid when supported by consistent-polarity attachment grouping (Experiments $2 \mathrm{~A}, 3 \mathrm{~A}, 4 \mathrm{~A}, 5 \mathrm{~A}, 5 \mathrm{C}$, $6 \mathrm{~A}, 7 \mathrm{~A}$, and $7 \mathrm{C}$ ) because, when a low-pass input was analyzed, it was generally easy to distinguish a larger region from individual half-size regions. Search remained quite efficient when it was supported by consistent-polarity contour grouping (Experiments 2B, 3B, 4B, 6B, 7B, and $7 \mathrm{D})$; across the gap of a contour group, nearby elements generally merged together and formed a mildly connected region. When a consistent-polarity attachment group was searched for among consistent-polarity contour groups
(Experiment 2D), the two kinds of group were distinguishable in terms of their overall luminance level, especially near the central gap region, and so still supported fairly efficient search. When a misaligned group (Experiment 2C), opposite-polarity groups (Experiments 3C, 3D, $4 \mathrm{C}, 4 \mathrm{D}, 5 \mathrm{~B}, 5 \mathrm{D}, 6 \mathrm{C}$, and $6 \mathrm{D}$ ), or misaligned oppositepolarity groups (Experiments $3 \mathrm{E}$ and $4 \mathrm{E}$ ) were searched for, the targets and distractors were generally difficult to distinguish. In a low-pass image, a misaligned group was highly confusable with two nearby individual distractors, and the opposite-polarity elements of an opposite-polarity group were not merged naturally to form a single perceptual region. Therefore, search for a target in these conditions was not supported by identifying a larger region from individual small regions within a single contrast sign; rather, search became fairly inefficient, because each item had to be checked for a complement. When an opposite-polarity attachment group was searched for among opposite-polarity contour groups (Experiments $3 \mathrm{~F}$ and $4 \mathrm{~F}$ ), search became highly inefficient, because, in such a situation, search could not be done even by checking for a complement, since each distractor contained a complement as well. In this case, search had to rely on a high-pass mechanism that was capable of identifying whether a gap was present within an item. The operation of a high-pass mechanism was conceivably serial and required spatial attention.

The similarity between the performance patterns of attachment and contour grouping suggests that attachment and contour grouping may have their basis in a common mechanism. Elder and Zucker (1993) found that search time for a contour group decreased smoothly when the central gap of a contour group was reduced in its size and gradually became an attachment group. The absence of a dramatic drop in search time when the contour group was about to become an attachment group was consistent with this suggestion.

Taken together, the present findings generally suggest that perceptual grouping occurs most efficiently when a group of visual elements is attached and is consistent in their contrast polarity. This is consistent with some theoretical formulations (e.g., Palmer \& Rock, 1994) that regard attached regions as entry-level units for perceptual grouping. However, attention is generally required for putting together separated elements, such as nearby elements with aligned contours. These grouping processes operate only on inputs that have the same contrast sign. In contrast, more attention is required to put together visual elements with opposite contrast polarity. Selection of elements that vary in contrast polarity seems to be based on fairly general processes, such as proximity-based grouping or attentional selection of multiple stimuli.

\section{AUTHOR NOTE}

This research was supported by an HKU Postgraduate Fellowship to L.K.H.C., and by Grant (HKU 7649/06H) from the Hong Kong Research Grants Council to W.G.H. We thank Ron Rensink for his helpful comments. Correspondence concerning this article should be addressed to L. K. H. Chan, Department of Psychology, University of Hong Kong, Pokfulam, Hong Kong (e-mail: clouis@graduate.hku.hk). 


\section{REFERENCES}

Broadbent, D. E. (1958). Perception and communication. New York: Pergamon.

Chun, M. M., \& Wolfe, J. M. (1996). Just say no: How are visual searches terminated when there is no target present? Cognitive Psychology, 30, 39-78.

Duncan, J., \& Humphreys, G. W. (1989). Visual search and stimulus similarity. Psychological Review, 96, 433-458.

Elder, J. H., \& ZuCKER, S. W. (1993). The effect of contour closure on the rapid discrimination of two-dimensional shapes. Vision Research, 33, 981-991.

Gilchrist, I. D., Humphreys, G. W., Riddoch, M. J., \& Neumann, H. (1997). Luminance and edge information in grouping: A study using visual search. Journal of Experimental Psychology: Human Perception \& Performance, 23, 464-480.

Grossberg, S., \& Mingolla, E. (1985). Neural dynamics of form perception: Boundary completion, illusory figures, and neon color spreading. Psychological Review, 92, 173-211.

Kellman, P. J., \& Shipley, T. F. (1991). A theory of visual interpolation in object perception. Cognitive Psychology, 23, 141-221.

NeIsser, U. (1967). Cognitive psychology. New York: AppletonCentury-Crofts.

PALmer, S., \& Rock, I. (1994). Rethinking perceptual organization: The role of uniform connectedness. Psychonomic Bulletin \& Review, 1, 29-55.

Rensink, R. A., \& EnNs, J. T. (1995). Preemption effects in visual search: Evidence for low-level grouping. Psychological Review, 102, 101-130.

Spehar, B. (2002). The role of contrast polarity in perceptual closure. Vision Research, 42, 343-350.
Treisman, A. [M.] (1988). Features and objects: The fourteenth Bartlett memorial lecture. Quarterly Journal of Experimental Psychology, 40A, 201-237

Treisman, A., M., \& Gelade, G. (1980). A feature-integration theory of attention. Cognitive Psychology, 12, 97-136.

\section{NOTES}

1. We always refer to search efficiency of target-present trials in this article, unless otherwise specified.

2. On each display, cells were defined in the following way: $n$ concentric circles with radii $n \times r$ defined 1 circle and $n-1$ rings. The radius of the outermost ring (the outer boundary) was always 288 pixels (subtending $11.7^{\circ}$ ), and thus the value $r$ equals $288 / n$. $2 n-1$ equal sections were defined in each $n$th ring, with the inner circle as the first "ring." For $s$ cells, $\sqrt{ } s$ rings were defined, and thus $n=\sqrt{ } s$. We always chose a value for $s$ such that its square root was an integer. Each region defined in the display was of equal area.

3. It should be noted that although we have some evidence showing that contour alignment fails to facilitate grouping across opposite contrast polarity, we do not have such evidence for attachment. Therefore, all we can say is that attachment grouping requires more attention when it operates across contrast polarity.

4. We thank Ron Rensink for pointing out this possibility.

5. This analysis shows that our data are explained fairly well by a simple spatial-filtering mechanism. However, there are demonstrably grouping types that are not explained by spatial filtering (e.g., Rensink \& Enns, 1995, p. 123).

(Manuscript received March 28, 2008; revision accepted for publication April 4, 2009.) 